\title{
A NATURAL EXTENSION OF NATURAL DEDUCTION
}

\author{
PETER SCHROEDER-HEISTER ${ }^{1}$
}

One of the main ideas of calculi of natural deduction, as introduced by Jaśkowski and Gentzen, is that assumptions may be discharged in the course of a derivation. As regards sentential logic, this conception will be extended in so far as not only formulas but also rules may serve as assumptions which can be discharged. The resulting calculi and derivations with rules of any finite level are informally introduced in $\S 1$, while $\$ \S 2$ and 3 state formal definitions of the concepts involved and basic lemmata. Within this framework, a standard form for introduction and elimination rules for arbitrary $n$-ary sentential operators is motivated in $\$ 4$, understood as a contribution to the theory of meaning for logical signs. $\S 5$ proves that the set $\{\&, \vee, \supset, \curlywedge\}$ of standard intuitionistic connectives is complete, i.e. \&, $\vee, \supset$ and $\curlywedge$ suffice to express each $n$-ary sentential operator having rules of the standard form given in $\$ 4$. $\$ 6$ makes some remarks on related approaches. For an extension of the conception presented here to quantifier logic, see [11].

§1. Derivations with rules of higher levels-informal exposition. Assumptions in sentential calculi technically work like additional axioms. A formula $\alpha$ is derivable from formulas $\beta_{1}, \ldots, \beta_{n}$ in a calculus $\mathscr{C}$ if $\alpha$ is derivable in the calculus $\mathscr{C}^{\prime}$ resulting from $\mathscr{C}$ by adding $\beta_{1}, \ldots, \beta_{n}$ as axioms. But whereas "genuine" axioms belong to the chosen framework and are usually assumed to be valid in some sense, assumptions bear an ad hoc character: they are considered only within the context of certain derivations. When deriving $\alpha$ from $\beta_{1}, \ldots, \beta_{n}$ we do not want to change our framework and to extend the calculus $\mathscr{C}$; we are interested in the derivability relation between $\beta_{1}, \ldots, \beta_{n}$ and $\alpha$ with respect to $\mathscr{C}$. This ad hoc character of assumptions, as compared with axioms, is made obvious in natural deduction systems: some of their inference rules allow one to discharge assumptions used in the derivations of the premises - that means, such assumptions are used only in specific subderivations for the purpose of establishing a certain formula in the superior derivation. Whereas inference rules of a Hilbert-type system may be written as

$$
\frac{\beta_{1} \cdots \beta_{n}}{\alpha}
$$

Received April 1, 1982; revised August 31, 1983.

${ }^{1}$ I should like to thank Professors G. Hasenjaeger and D. Prawitz for their support and criticism of my dissertation, and the anonymous referee (to whom the title of this paper is also due) for helpful suggestions. 
inference rules in a calculus of natural deduction can be given as

$$
\frac{\frac{\Gamma_{1}}{\beta_{1}} \cdots \frac{\Gamma_{n}}{\beta_{n}}}{\alpha}
$$

where the $\Gamma$ 's are finite lists of formulas representing the assumptions which may be discharged within the subderivations of the corresponding $\beta$ 's by the application of this rule. A rule of form (1) can be considered to be a formula tree (growing upwards) of height 2 , a rule of form (2) to be a formula tree of height 3 (provided not all $\Gamma$ 's are empty).

Instead of considering only ad hoc axioms (i.e. assumption formulas) we can also regard ad hoc inference rules, that is, inference rules of form (1) used as assumptions. Assumption rules technically work like additional basic rules: $\alpha$ is derivable from assumption formulas $\beta_{1}, \ldots, \beta_{n}^{*}$ and assumption rules $\rho_{1}, \ldots, \rho_{m}$ in $\mathscr{C}$ if $\alpha$ is derivable in $\mathscr{C}^{\prime}$, where $\mathscr{C}^{\prime}$ results from $\mathscr{C}$ by adding $\beta_{1}, \ldots, \beta_{n}$ as axioms and $\rho_{1}, \ldots, \rho_{m}$ as basic inference rules. By "basic rule" or "basic inference rule" I mean rules which constitute, together with axioms, the derivability concept of a calculus. Certain rules are the basic rules of a calculus in the same sense as certain formulas are its axioms.

Consider for example the abstract calculus whose formulas are finite strings consisting of the atoms + and $O$. Let it have as basic rules all instances of

$$
\begin{array}{lll}
(\mathscr{R} 1) & \frac{+X}{X+} & (\mathscr{R} 2) \frac{X \quad Y \circ}{X Y \circ}
\end{array}
$$

where $X$ and $Y$ are schematic letters for formulas. Then

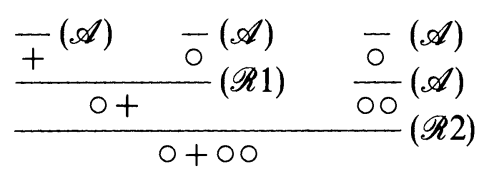

denotes a derivation of $\bigcirc+\circ \circ$ from the assumptions,$+ \circ$ (assumption formulas) and $\frac{O}{0 O}$ (assumption rule). To the right of the lines it is indicated whether the formula immediately below the line is an assumption formula or obtained by application of an assumption rule $(\mathscr{A})$, or whether it is obtained by application of a basic rule that is an instance of $\mathscr{R} 1$ or $\mathscr{R} 2$. These indications however are considered to be only metalinguistic comments, and are not part of the notation itself.

The idea of assumption rules leads to an extension of natural deduction if we introduce inference rules allowing one to discharge assumption rules as well as assumption formulas. Such rules can be presented in form (2) where the $\Gamma$ 's may now contain rules of form (1) besides formulas; they may be considered formula trees of height 4 (provided at least one of the $\Gamma$ 's contains a rule of form (1)). Examples are the instances of the schema

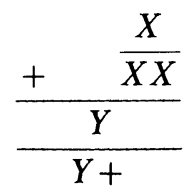


If we extend the above calculus by this schema, the following denotes a derivation of $\circ+\circ \circ+$ from $\circ:$

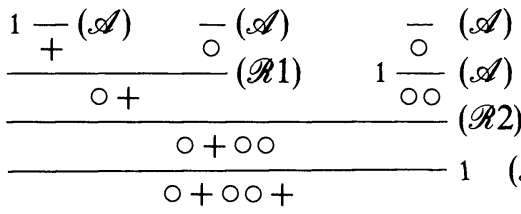

Here the small numeral (which does belong to the notation of the derivation) on the left-hand side of a line denotes that the formula occurrence immediately below it (if it is an assumption formula) or the application of the assumption rule indicated by the line is discharged by the application of the rule which is indicated by a line having the same numeral on its right-hand side. In the present case + and $\frac{\mathrm{O}}{\mathrm{OO}}$ are discharged by application of

$$
\frac{+\frac{0}{00}}{\frac{0+00}{0+00+}}
$$

which is an instance of $\mathscr{R} 3$. A derivation depends on those assumption formulas occurring somewhere without a numeral to the left of the line immediately above it and on those assumption rules being applied somewhere without a numeral to the left of the line indicating this application. Both cases can be put into one if we consider assumption formulas $\alpha$ to be result of the application of an assumption rule $\bar{\alpha}$ without premises. So from now on we shall use "assumption" and "assumption rule" synonymously.

This procedure can be iterated: we may use rules of form (2) as assumption rules which are dischargeable by the application of rules of higher complexity, and so on. Rules are then arbitrary finite formula trees (growing upwards); their height will be called the level of the rule. This leads to a concept of derivation where all kinds of inference rules may be used as assumptions and may be discharged by the application of other rules. In particular, assumptions may be discharged by the application of assumption rules.

So formulas $\alpha$ are rules of level 1 (allowing one to infer $\alpha$ without premises), rules of form (1) are rules of level 2, rules allowing one to discharge formulas (like つintroduction and $\vee$-elimination in natural deduction systems) are rules of level 3 , rules allowing one to discharge rules of level 2 are rules of level 4 etc.

Examples of rules of level 5 are instances of the schema

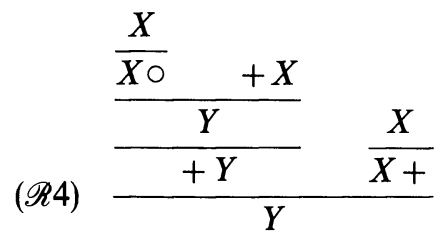

Extending the preceding calculus with the instances of $\mathscr{R} 4$ as basic rules, the 
following denotes a derivation of $\circ+0+$ from $+\circ$ :

$$
\begin{aligned}
& 1 \frac{-(\mathscr{A})}{0} \\
& 3 \frac{\bar{O}^{\circ}}{(\mathscr{A})} \quad \overline{+0}(\mathscr{A}) \\
& 3+(\mathscr{A}) \\
& 2-1 \quad(\mathscr{A}) \\
& +0+0 \\
& \frac{0+0}{0+0+} 3
\end{aligned}
$$

As the numerals to the left and to the right of the line above the topmost occurrence of $\circ+\circ$ show, the application of the assumption rule

$$
\frac{\frac{0}{00}+0}{0+0}
$$

discharging the assumption formula $\bigcirc$ is itself discharged by application of an instance of $\mathscr{R} 4$.

In general, the meaning of a rule of form (2) can be informally stated as: If for each $i(1 \leq i \leq n) \beta_{i}$ has been derived using certain assumption rules, one may immediately infer $\alpha$ and for each $i$ discharge assumption rules of $\Gamma_{i}$ on which the derivation of $\beta_{i}$ depends.

We do not require assumptions which could be discharged by the application of a rule to be actually used in a derivation. For example,

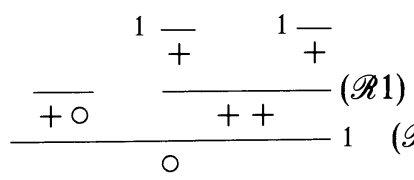

denotes a derivation of $\circ$ from $+\circ$ applying the instance

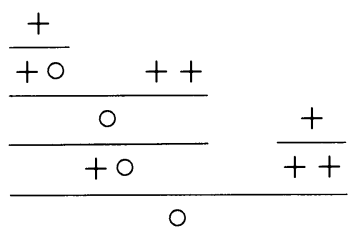

of $\mathscr{R} 4$, although the weaker rule

$$
\begin{aligned}
& +\frac{t}{t+} \\
& 0
\end{aligned}
$$

would have been sufficient to establish this derivation. Furthermore, in which order the assumption rules which may be discharged (i.e. the members of the $\Gamma$ 's in (2)) are 
stated is not relevant for the application of this rule in a derivation. In order to capture this we shall define the notion of a subrule of a rule in such a way that each application of a subrule can be considered to be an application of the rule itself (e.g., (4) will denote a subrule of (3)).

Moreover, an assumption that is actually used in a derivation and could be discharged by the application of a rule, need not be discharged at certain places or at any places at all. So

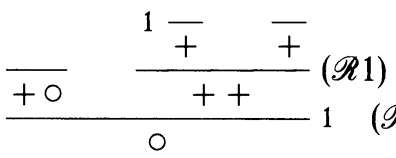

denotes a derivation of $\circ$ from $+\circ$ and + . This point will be included in our definitions by adopting Prawitz's [7] technique of "discharge functions" which correspond to our numerals and state which assumption is considered discharged by which rule application.

§2. Rules and derivations - basic definitions. In this and the following section we leave unspecified what is counted as a formula.

Definition 2.1. A rule is a finite formula tree growing upwards. A tree is here considered to be a graphical arrangement, not an abstract object. Speaking of formulas occurring (immediately) below or above others, of top formulas and the downmost or end formula etc. is thus explained. The height of the tree (i.e. the length of its longest branch) is called the level of the rule.

From now on we use, if not stated otherwise, $\alpha, \beta$, and $\gamma$ as syntactical variables for formulas, $\rho$ for rules (which include formulas as a limiting case) and $\Gamma$ and $\Delta$ for lists of rules (all with and without primes and indices). Lists are linear graphical arrangements of signs called their members (including the empty list).

DEFinition 2.2. Subrules are defined as follows: Rules of level $\leq 2$ have only themselves as subrules. A rule

$$
\frac{\frac{\Gamma_{1}^{\prime}}{\beta_{1}} \cdots \frac{\Gamma_{n}^{\prime}}{\beta_{n}}}{\alpha}
$$

is a subrule of

$$
\frac{\frac{\Gamma_{1}}{\beta_{1}} \cdots \frac{\Gamma_{n}}{\beta_{n}}}{\alpha}
$$

if for all $i(1 \leq i \leq n) \Gamma_{i}^{\prime}$ results from $\Gamma_{i}$ by arbitrarily often performing the operations of omitting, duplicating and reordering members or replacing members by subrules of them.

One could (but we do not) identify a rule with the set of its subrules (that would make rules which are subrules of each other identical).

Prawitz's concept of discharge functions is modified somewhat for our purposes. Discharge functions have as arguments not only top formulas of a formula tree. 
Definition 2.3. A discharge function for a finite formula tree $\mathscr{T}$ is a function $f$ defined on the set of all formula occurrences of $\mathscr{T}$ such that $f(\alpha)$ is either $\alpha$ or a formula occurrence below $\alpha$.

Discharge functions are represented in the notation for derivations by small numerals. If $f(\alpha)=\beta$ then $\alpha$ or the assumption rule whose conclusion is $\alpha$ is discharged by the application of the rule one of whose premises is $\beta$, if there is such a rule. Otherwise $\beta$ is the downmost formula in $\mathscr{T}$, and the assumption rule with the conclusion $\alpha$ is not discharged.

Definition 2.4. A derivation is a pair $(\mathscr{T}, f)$ consisting of a finite formula tree $\mathscr{T}$ together with a discharge function $f$ for $\mathscr{T}$.

This definition does not refer to any basic inference rule. We can define a rule assignment $g$ for a derivation $(\mathscr{T}, f)$, associating with each formula occurrence $\alpha$ of $\mathscr{T}$ a rule $g(\alpha)$ in such a way that $\alpha$ can be considered to be the conclusion of an application of $g(\alpha)$ (or of any rule of which $g(\alpha)$ is a subrule). If no basic rules are given, $(\mathscr{T}, f)$ may then be taken as a derivation of its downmost formula $\gamma$ from assumption rules $g\left(\beta_{1}\right), \ldots, g\left(\beta_{n}\right)$, where $\beta_{1}, \ldots, \beta_{n}$ are those formula occurrences in $\mathscr{T}$ which are neither discharged nor conclusions of discharged assumptions; that is, $f\left(\beta_{i}\right)=\gamma(1 \leq i \leq n)$. So there are nontrivial derivations using only assumption rules and no basic rules.

Definition 2.5. $g$ is a rule assignment for a derivation $(\mathscr{T}, f)$ iff $g$ associates with each formula occurrence $\alpha$ of $\mathscr{T}$ a rule $g(\alpha)$ such that if $\alpha$ is a topmost formula, then $g(\alpha)$ is $\alpha$, and if $\alpha$ occurs in $\mathscr{T}$ as

$$
\begin{gathered}
\vdots \\
\frac{\beta_{1} \cdots \beta_{n}}{\alpha}
\end{gathered}
$$

then $g(\alpha)$ is

$$
\frac{\frac{\Gamma_{1}}{\beta_{1}} \cdots \frac{\Gamma_{n}}{\beta_{n}}}{\alpha}
$$

where for all $i(1 \leq i \leq n) \Gamma_{i}$ contains exactly the rules $g(\gamma)$ for all those $\gamma$ such that $f(\gamma)=\beta_{i}$.

Obviously, a rule assignment always exists, and, for two rule assignments $g_{1}, g_{2}$ for $(\mathscr{T}, f)$ and any $\alpha, g_{1}(\alpha)$ and $g_{2}(\alpha)$ can differ only in the order of the members of the $\Gamma$ 's and are subrules of each other. So a specific choice of a rule assignment is not relevant for the following.

Now we assume that certain rules may be distinguished as basic rules.

Definition 2.6. A derivation $(\mathscr{T}, f)$ having $\alpha$ as downmost formula is a derivation of $\alpha$ from $\Delta$ iff there is a rule assignment $g$ for $(\mathscr{T}, f)$ such that, for each formula occurrence $\beta$ of $\mathscr{T}$, if $f(\beta)=\alpha$, then $g(\beta)$ is a subrule of a member of $\Delta$ or of a basic rule. $\alpha$ is derivable from $\Delta(\Delta \vdash \alpha)$ iff there is a derivation of $\alpha$ from $\Delta$.

If we speak of a derivation or of derivability from $\rho_{1}, \ldots, \rho_{n}$, we mean the list $\rho_{1} \cdots \rho_{n}$, and by $\Gamma, \Delta$ or $\alpha, \Delta$ we mean the lists formed by connecting $\Gamma$ and $\Delta$ or $\alpha$ and $\Delta$, and so on. 
If one wants to have a decidable derivation relation, one must require of the set of basic rules that "subrule of a basic rule" be decidable. If we restrict the arguments of a discharge function only to top formulas, Definition 2.6 includes as a special case the definition of derivability for a natural deduction system of the Gentzen-Prawitz style.

When speaking about rules we shall sometimes use a linear notation like $\left\langle\rho_{1}, \ldots, \rho_{n}\right\rangle \Rightarrow \alpha, \Delta \Rightarrow \alpha$ and $\left\langle\Gamma_{1} \Rightarrow \beta_{1}, \ldots, \Gamma_{n} \Rightarrow \beta_{n}\right\rangle \Rightarrow \alpha$ for rules

$$
\frac{\rho_{1} \cdots \rho_{n}}{\alpha}, \quad \frac{\Delta}{\alpha}, \quad \frac{\frac{\Gamma_{1}}{\beta_{1}} \cdots \frac{\Gamma_{n}}{\beta_{n}}}{\alpha}
$$

respectively. If $\rho$ is of the form

$$
\frac{\rho_{1} \cdots \rho_{n}}{\alpha}
$$

we denote by $(\rho)_{1}$ the system $\rho_{1} \cdots \rho_{n}$ of the premises of $\rho$, and by $(\rho)_{2}$ the conclusion $\alpha .(\rho)_{1}$ is the empty system if $\rho$ is a formula.

One could consider rules written linearly with $\Rightarrow$ to be object language expressions and in a derivation state assumption rules e.g. to the left of the line where it is applied, as done in Schroeder-Heister [9]. Assumption rules would then be part of a derivation itself and would function like a kind of implication which is to be distinguished from the sentential connective of implication $\supset$, and which may be iterated only to the left. This would however lead, if one wants to consider derivations to be representations of arguments, to the question in what sense rules can be parts of an argument itself (cf. Schroeder-Heister [10]) - a problem which is avoided here. Our rules are rules in the genuine sense.

\section{\$3. The derivability of rules. Basic lemmata.}

Definition 3.1. A rule $\left\langle\Gamma_{1} \Rightarrow \beta_{1}, \ldots, \Gamma_{n} \Rightarrow \beta_{n}\right\rangle \Rightarrow \alpha$ is derivable from a list of rules $\Gamma$, if for all $\Delta$ the following holds: if for all $i(1 \leq i \leq n) \Gamma, \Delta, \Gamma_{i} \vdash \beta_{i}$, then $\Gamma, \Delta \vdash \alpha$.

This definition follows the meaning we have assigned to a rule. However, derivability should be something that can be established by a derivation itself and not only by a procedure transforming derivations into derivations. The following lemma shows that this is the case.

Lemma 3.2. $\left\langle\Gamma_{1} \Rightarrow \beta_{1}, \ldots, \Gamma_{n} \Rightarrow \beta_{n}\right\rangle \Rightarrow \alpha$ is derivable from $\Gamma$ iff $\Gamma, \Gamma_{1} \Rightarrow$ $\beta_{1}, \ldots, \Gamma_{n} \Rightarrow \beta_{n} \vdash \alpha$ (or shorter: $\rho$ is derivable from $\Gamma$ iff $\left.\Gamma,(\rho)_{1} \vdash(\rho)_{2}\right)$.

The proof of this lemma uses three other lemmata for which we need some notational conventions. We shall use $\Gamma \vdash \rho$ as an abbreviation for $\Gamma,(\rho)_{1} \vdash(\rho)_{2}$. $\Gamma \vdash \Delta$ means that $\Gamma \vdash \rho$ for each member $\rho$ of $\Delta$. In particular, if $\Delta$ is empty, $\Gamma \vdash \Delta$ is true. $\Gamma$ H $\Delta$ means that $\Gamma \vdash \Delta$ and $\Delta \vdash \Gamma$.

Lemma 3.3. If $\Delta \vdash \alpha$, then $\Gamma, \Delta \vdash \alpha$.

Lemma 3.4. $\rho \vdash \rho$, i.e. $\rho,(\rho)_{1} \vdash(\rho)_{2}$.

Proof (by induction on the level of $\rho$ ). If it is 1 , we have to prove $\alpha \vdash \alpha$, which is trivial. If it is 2 , we have to prove $\left\langle\beta_{1}, \ldots, \beta_{n}\right\rangle \Rightarrow \alpha, \beta_{1}, \ldots, \beta_{n} \vdash \alpha$, which is trivial as well. If it is $m>2$, then $\rho$ is of the form $\left\langle\Gamma_{1} \Rightarrow \beta_{1}, \ldots, \Gamma_{n} \Rightarrow \beta_{n}\right\rangle \Rightarrow \alpha$, where for at least one $i(1 \leq i \leq n) \Gamma_{i}$ is nonempty. By the induction hypothesis we have for all those $i: \Gamma_{i} \Rightarrow \beta_{i}, \Gamma_{i} \vdash \beta_{i}$ (since $\Gamma_{i} \Rightarrow \beta_{i}$ is of level $<m$ ); thus by Lemma $3.3(\rho)_{1}, \Gamma_{i} \vdash \beta_{i}$ for all $i$ 
$(1 \leq i \leq n)$, i.e. we have derivations of $\beta_{i}$ from $(\rho)_{1}$ and $\Gamma_{i}$. From it we obtain a derivation of $\alpha$ from $(\rho)_{1}$ and $\rho$ by application of $\rho$.

LEMMA 3.5. If $\Delta \vdash \rho$ and $\Delta, \rho \vdash \gamma$, then $\Delta \vdash \gamma$.

ProOF (by induction on the level of $\rho$ ). If it is 1 , we have to prove that if $\Delta \vdash \alpha$ and $\Delta, \alpha \vdash \gamma$ then $\Delta \vdash \gamma$, which is trivial. If it is 2 , we have to prove that if $\Delta, \beta_{1}, \ldots, \beta_{n} \vdash \alpha$ and $\Delta,\left\langle\beta_{1}, \ldots, \beta_{n}\right\rangle \Rightarrow \alpha \vdash \gamma$ then $\Delta \vdash \gamma$. We replace each application of $\left\langle\beta_{1}, \ldots, \beta_{n}\right\rangle \Rightarrow \alpha$ in the given derivation of $\gamma$ from $\Delta$ and $\left\langle\beta_{1}, \ldots, \beta_{n}\right\rangle \Rightarrow \alpha$ by the given derivation of $\alpha$ from $\Delta$ and $\beta_{1}, \ldots, \beta_{n}$. If the level of $\rho$ is $m>2$, then $\rho$ is of the form $\left\langle\Gamma_{1} \Rightarrow \beta_{1}, \ldots, \Gamma_{n} \Rightarrow \beta_{n}\right\rangle \Rightarrow \alpha$ where at least one $\Gamma_{i}$ is nonempty. Consider a topmost occurrence of $\alpha$ in the given derivation $(\mathscr{T}, f)$ of $\gamma$ from $\Delta$ and $\rho$ such that $\alpha$ is the conclusion of an application of $\rho$ (that is, $f(\alpha)=\gamma$ and $g(\alpha)$ is a subrule of $\rho$ for a rule assignment $g$ for $(\mathscr{T}, f)$ ). Then there are derivations of $\beta_{i}$ from $\Delta, \Gamma_{i}$ and $\Delta_{i}$ for each $i(1 \leq i \leq n)$, where the $\Delta_{i}$ are lists of assumption rules which are discharged in $(\mathscr{T}, f)$ by applications of rules below $\alpha$. That is, we have $\Delta, \Gamma_{i}, \Delta_{i} \vdash \beta_{i}$, which is the same as $\Delta, \Delta_{i} \vdash \Gamma_{i} \Rightarrow \beta_{i}$. Since $\Gamma_{i} \Rightarrow \beta_{i}$ is of level $<m$, we obtain by $n$-fold application of the induction hypothesis to $\Delta, \Delta_{i} \vdash \Gamma_{i} \Rightarrow \beta_{i}$ and to $\Delta \vdash \rho$ (i.e. $\Delta$, $\left.\Gamma_{1} \Rightarrow \beta_{1}, \ldots, \Gamma_{n} \Rightarrow \beta_{n} \vdash \alpha\right)$ a derivation of $\alpha$ from $\Delta$ and $\Delta_{i}$. Replacing the considered application of $\rho$ in $(\mathscr{T}, f)$ by the derivation of $\alpha$ from $\Delta$ and $\Delta_{i}$ we obtain a derivation $\left(\mathscr{T}^{\prime}, f^{\prime}\right)$ of $\gamma$ from $\Delta$ and $\rho$ which has at least one application of $\rho$ less than $(\mathscr{T}, f)$. (The $\Delta_{i}$ are discharged in $\left(\mathscr{T}^{\prime}, f^{\prime}\right)$ below the considered $\alpha$ as in $(\mathscr{T}, f)$.) Successive application of this procedure yields the assertion.

ProOF OF LeMma 3.2. By Lemmata 3.3. and 3.4 we have $\Gamma, \Gamma_{1} \Rightarrow \beta_{1}, \ldots, \Gamma_{n} \Rightarrow \beta_{n}$, $\Gamma_{i} \vdash \beta_{i}$ for all $i(1 \leq i \leq n)$. Thus, if $\left\langle\Gamma_{1} \Rightarrow \beta_{1}, \ldots, \Gamma_{n} \Rightarrow \beta_{n}\right\rangle \Rightarrow \alpha$ is derivable from $\Gamma$, then $\Gamma, \Gamma_{1} \Rightarrow \beta_{1}, \ldots, \Gamma_{n} \Rightarrow \beta_{n} \vdash \alpha$. Conversely, if $\Gamma, \Gamma_{1} \Rightarrow \beta_{1}, \ldots, \Gamma_{n} \Rightarrow \beta_{n} \vdash \alpha$, then from the hypotheses $\Gamma, \Delta, \Gamma_{i} \vdash \beta_{i}(1 \leq i \leq n)$, which are the same as $\Gamma, \Delta \vdash \Gamma_{i} \Rightarrow \beta_{i}$, and by $n$-fold application of Lemmata 3.3 and 3.5 we obtain $\Gamma, \Delta \vdash \alpha$, i.e. $\left\langle\Gamma_{1} \Rightarrow \beta_{1}, \ldots, \Gamma_{n} \Rightarrow \beta_{n}\right\rangle \Rightarrow \alpha$ is derivable from $\Gamma$.

In view of Lemma 3.2 we are justified to identify $\Gamma \vdash \rho$ with the derivability of $\rho$ from $\Gamma$.

Definition 3.6. A component of a rule $\rho$ is a rule $\rho^{\prime}$ that is a subtree of $\rho$. An element of $\rho$ is the downmost formula of a subtree of $\rho$ (i.e. one of the formula occurrences from which $\rho$ is built up).

LEMMA 3.7. Let $\rho^{\prime}$ t $\rho^{\prime \prime}$. Let $\rho^{\prime}$ occur as a component of $\rho$ and $\rho\left[\rho^{\prime} / \rho^{\prime \prime}\right]$ be the result of replacing this component by $\rho^{\prime \prime}$. Then $\rho$ t $\rho\left[\rho^{\prime} / \rho^{\prime \prime}\right]$.

ProOF (by induction on the level of $\rho$ ). If $\rho$ is identical with $\rho^{\prime}$, the assertion is obvious. (This is in particular the case if $\rho$ has level 1.) Otherwise $\rho$ is $\left\langle\rho_{1}, \ldots, \rho_{n}\right\rangle \Rightarrow \beta$. $\rho^{\prime}$ must be a component of a $\rho_{i}(1 \leq i \leq n)$, and by the induction hypothesis we have for this $\rho_{i}: \rho_{i}$ t $\rho_{i}\left[\rho^{\prime} / \rho^{\prime \prime}\right]$. By Lemmata 3.4 and 3.5 we obtain $\rho$, $\rho_{1}, \ldots, \rho_{i}\left[\rho^{\prime} / \rho^{\prime \prime}\right], \ldots, \rho_{n} \vdash \beta$, i.e. $\rho \vdash \rho\left[\rho^{\prime} / \rho^{\prime \prime}\right]$, and analogously $\rho\left[\rho^{\prime} / \rho^{\prime \prime}\right] \vdash \rho$.

LEMMA 3.8. Let $\alpha+1 \beta$. Let $\alpha$ be an element of $\rho$ and let $\rho[\alpha / \beta]$ result from $\rho$ by replacing this element in $\rho$ by $\beta$. Then $\rho \Vdash \rho[\alpha / \beta]$.

PROOF. If $\rho$ is not identical with $\alpha$ (in which case the assertion is trivial), $\rho$ contains $\left\langle\rho_{1}, \ldots, \rho_{n}\right\rangle \Rightarrow \alpha$ as a component. Replacing $\left\langle\rho_{1}, \ldots, \rho_{n}\right\rangle \Rightarrow \alpha$ by $\left\langle\rho_{1}, \ldots, \rho_{n}\right\rangle \Rightarrow \beta$ is the same as replacing $\alpha$ by $\beta$. Since from $\alpha+\beta$ we can by Lemmata 3.4 and 3.5 infer

$$
\left\langle\rho_{1}, \ldots, \rho_{n}\right\rangle \Rightarrow \alpha \Vdash\left\langle\rho_{1}, \ldots, \rho_{n}\right\rangle \Rightarrow \beta,
$$

the assertion follows from Lemma 3.7. 
§4. Sentential calculi for $n$-ary operators. We present a framework for the treatment of arbitrarily many sentential operators (in short: operators) of any number of argument places. That means, we do not present a specific calculus, but describe a language for a generalized sentential logic leaving the sentential operators themselves unspecified, and motivate a standard form for basic rules for these operators (again leaving the rules themselves unspecified). This standard form is justified by arguing that rules of this form give a meaning to the operator involved, i.e. we describe a semantical framework in the sense of the theory of meaning.

We assume finitely or denumerably many sentential operators to be given, each with an associated natural number $\geq 0$ as the number of its argument places, and furthermore infinitely many sentence letters. We use $S$ and $S^{\prime}$ as syntactical variables for operators. All sentence letters are atomic formulas. Formulas are atomic formulas and expressions $S \alpha_{1} \cdots \alpha_{n}$ where $S$ is an $n$-ary operator and the $\alpha_{1}, \ldots, \alpha_{n}$ are formulas (in particular, $S$ is a formula if $S$ is 0 -ary) If $S$ is binary, $\left(\alpha_{1} S \alpha_{2}\right)$ may be written instead of $S \alpha_{1} \alpha_{2}$, where outer brackets may be omitted. Subformulas of a formula are the formula itself and, if it is $S \alpha_{1} \cdots \alpha_{n}$, the subformulas of $\alpha_{1}, \ldots, \alpha_{n}$. Subformulas of a rule are defined as subformulas of its elements, subformulas of a list of rules as subformulas of its members.

Furthermore, we assume infinitely many schematic letters for formulas be given. Syntactical variables for schematic letters are $A, B$, and $C$, with and without indices. Formula and rule schemata are built up from schematic letters in the same way as formulas and rules are built up from sentence letters, i.e., formula schemata are schematic letters and expressions $S F_{1} \cdots F_{n}$, where $F_{1}, \ldots, F_{n}$ are formula schemata. Rule schemata are finite trees of formula schemata. Formula and rule schemata are instantiated by replacing all schematic letters with formulas. Syntactical variables for formula schemata: $F$, for rule schemata: $R$, for lists of rule schemata: $\Phi$ (all with and without indices). (Remark. According to these definitions, we consider schemata to be formal objects. Schematic letters for formulas are distinguished from syntactical variables for formulas.)

In order to indicate that schemata $F, R, \Phi$ contain at most $A_{1}, \ldots, A_{n}$ as schematic letters, where the $A_{1}, \ldots, A_{n}$ are all different, we also write $F\left(A_{1}, \ldots, A_{n}\right)$, $R\left(A_{1}, \ldots, A_{n}\right), \Phi\left(A_{1}, \ldots, A_{n}\right)$. For formulas $\alpha_{1}, \ldots, \alpha_{n}, F\left(\alpha_{1}, \ldots, \alpha_{n}\right), R\left(\alpha_{1}, \ldots, \alpha_{n}\right)$, $\Phi\left(\alpha_{1}, \ldots, \alpha_{n}\right)$ are then the result of substituting $\alpha_{i}$ for $A_{i}$ for all $i(1 \leq i \leq n)$ in $F, R, \Phi$. We say that $R\left(A_{1}, \ldots, A_{n}\right)$ or $\Phi\left(A_{1}, \ldots, A_{n}\right)$ is derivable, if for all formulas $\alpha_{1}, \ldots, \alpha_{n}$ we have $\vdash R\left(\alpha_{1}, \ldots, \alpha_{n}\right)$ or $\vdash \Phi\left(\alpha_{1}, \ldots, \alpha_{n}\right)$.

We want to justify a standard form for introduction and elimination rules for an $n$ ary operator $S$. This is based on the concept of the common content of lists of rules.

DEFINITION 4.1. The common content of $\Gamma_{1}, \ldots, \Gamma_{m}$ is the set of all $\rho$ such that $\Gamma_{i} \vdash \rho$ for all $i(1 \leq i \leq m)$.

As a limiting case we allow $m$ to be 0 . In that case the common content of 0 lists of rules consists of all rules $\rho$. This case will lead to intuitionistic logic with its characteristic absurdity rule. The concept of common content is a generalization of the concept of the logical content of a formula as the set of its logical consequences, as used e.g. by Tarski and Carnap (but whose roots go back at least to Frege).

We now assume that, with each $n$-ary operator $S, m(m \geq 0)$ lists

$$
\Phi_{1}\left(A_{1}, \ldots, A_{n}\right), \ldots, \Phi_{m}\left(A_{1}, \ldots, A_{n}\right)
$$


of rule schemata are associated. The limiting case $m=0$ means that no list is associated with $S$. $S$ is then called a $\perp$-operator. Since the meaning of $S$ will be determined by reference to the $\Phi_{i}$ and thus to the operators occurring in the $\Phi_{i}$, their meaning must be presumed to be already given. So we require that it must be possible to order the sentential operators in a sequence $S_{1}, S_{2}, \ldots$ such that for each operator $S$ the operators occurring in the associated lists $\Phi_{1}, \ldots, \Phi_{m}$ of rule schemata precede $S$ in this enumeration (i.e., if $S$ is $S_{l}$, then no $S_{k}$ with $k \geq l$ may occur in a $\Phi_{i}$ ). Then we want the basic rules for $S$ just to guarantee that the following condition is fulfilled:

Condition 4.2. For all $\alpha_{1}, \ldots, \alpha_{n}, S \alpha_{1} \cdots \alpha_{n}$ expresses the common content of the $\Phi_{i}\left(\alpha_{1}, \ldots, \alpha_{n}\right)(1 \leq i \leq m)$, i.e. for all $\alpha_{1}, \ldots, \alpha_{n}$ and $\rho$ we have $S \alpha_{1} \cdots \alpha_{n} \vdash \rho$ iff for all $i(1 \leq i \leq m) \Phi_{i}\left(\alpha_{1}, \ldots, \alpha_{n}\right) \vdash \rho$.

This condition may be conceived as generalizing the aim of an explicit definition: If a formula $\alpha$ is explicitly defined by $\beta, \alpha$ has according to the definition the same content as $\beta$, since for all $\gamma$ it holds that $\alpha \vdash \gamma$ iff $\beta \vdash \gamma$. Another way of circumscribing Condition 4.2 is to say that for all $\alpha_{1}, \ldots, \alpha_{n}, S \alpha_{1} \cdots \alpha_{n}$ is a formula of maximal strength which can be inferred from $\Phi_{i}\left(\alpha_{1}, \ldots, \alpha_{n}\right)$ for all $i(1 \leq i \leq m)$ - which is very much like Tennant's "principle of harmony" [12, p. 74].

The reason for associating only lists of rule schemata with operators is the following: We want to leave open the possibility of considering our system of sentential logic as built up over a basic calculus (e.g. a Post system) generating valid atomic formulas, and the basic rules for operators should be independent of the choice of such a basic calculus (cf. Schroeder-Heister [9]).

Condition 4.2 leads to the following standard form of basic rule schemata for $S$ :

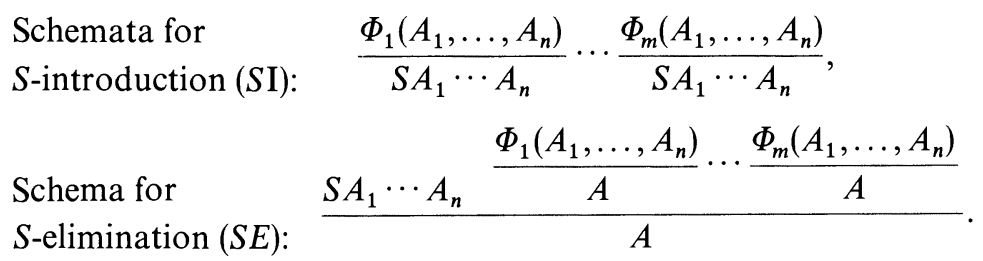

Here $A$ stands for a schematic letter different from $A_{1}, \ldots, A_{n}$. If $S$ is a $\perp$-operator, there is no $S$ I-rule and the schema for $S E$ is

$$
\frac{S A_{1} \cdots A_{n}}{A}
$$

TheOREM 4.3. Condition 4.2 is fulfilled iff SI and SE are derivable.

Proof. Let formulas $\alpha_{1}, \ldots, \alpha_{n}$ be given. From 4.2 it follows, taking $\rho$ to be $S \alpha_{1} \cdots \alpha_{n}$, that for all $i(1 \leq i \leq m)$

$$
\Phi_{i}\left(\alpha_{1}, \ldots, \alpha_{n}\right) \vdash S \alpha_{1} \cdots \alpha_{n},
$$

i.e. the derivability of $S$ I. Taking $\rho$ to be $\Gamma \Rightarrow \alpha$ for arbitrary $\Gamma$ and $\alpha$, we have that if for all $i(1 \leq i \leq m) \Phi_{i}\left(\alpha_{1}, \ldots, \alpha_{n}\right), \Gamma \vdash \alpha$, then $S \alpha_{1} \cdots \alpha_{n}, \Gamma \vdash \alpha$, i.e. the derivability of $S E$. Conversely, from $S \alpha_{1} \cdots \alpha_{n} \vdash \rho$ and $\Phi_{i}\left(\alpha_{1}, \ldots, \alpha_{n}\right) \vdash S \alpha_{1} \cdots \alpha_{n}$ for all $i(1 \leq i \leq m)$ we obtain $\Phi_{i}\left(\alpha_{1}, \ldots, \alpha_{n}\right) \vdash \rho$ for all $i(1 \leq i \leq m)$. Furthermore from $\Phi_{i}\left(\alpha_{1}, \ldots, \alpha_{n}\right) \vdash \rho$, 
i.e. $\Phi_{i}\left(\alpha_{1}, \ldots, \alpha_{n}\right),(\rho)_{1} \vdash(\rho)_{2}$, for all $i(1 \leq i \leq m)$, and the derivability of

$$
\left\langle S \alpha_{1} \cdots \alpha_{n}, \Phi_{1}\left(\alpha_{1}, \ldots, \alpha_{n}\right) \Rightarrow(\rho)_{2}, \ldots, \Phi_{m}\left(\alpha_{1}, \ldots, \alpha_{n}\right) \Rightarrow(\rho)_{2}\right\rangle \Rightarrow(\rho)_{2}
$$

we obtain $S \alpha_{1} \cdots \alpha_{n},(\rho)_{1} \vdash(\rho)_{2}$, i.e. $S \alpha_{1} \cdots \alpha_{n} \vdash \rho$.

The basic rules for the intuitionistic connectives $\&, \vee, \supset, \curlywedge$, as given in Prawitz [7], already have our standard form or can easily be transformed into it. The former holds for $\vee$ and $ᄉ$, whose basic rule schemata have the form

$$
(\vee \mathrm{I}) \frac{A}{A \vee B} \quad \frac{B}{A \vee B} \quad(\vee E) \frac{A \vee B \quad \frac{A}{B}}{C} \frac{B}{C}
$$

and

$$
\text { (人 E) } \frac{\text { 人 }}{A}
$$

The basic rule schemata for $\&$ can be written in the form

$$
\begin{array}{llll}
\text { (\& I) } \frac{A \quad B}{A \& B} & (\& \mathrm{E}) \frac{A \& B}{C} \frac{A}{C}
\end{array}
$$

since, obviously,

$$
\frac{A \& B}{A} \text { and } \frac{A \& B}{B}
$$

have both together the same strength as $\& \mathrm{E}$. The basic rule schemata for $\supset$ can be stated in the form

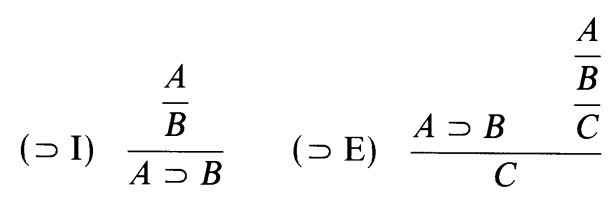

where $\supset \mathrm{E}$ is equivalent to the schema of modus ponens having the form

$$
\text { (MP) } \frac{A \supset B \quad A}{B}
$$

as the following lemma shows.

LEMMA 4.4. $M P$ is derivable iff $\supset E$ is derivable.

Proof. For formulas $\alpha, \beta, \gamma$, the derivation denoted by

$$
\frac{\frac{\overline{\alpha \supset \beta}(\mathscr{A}) \quad 1-(\mathscr{A})}{\beta}(\mathscr{A})}{\gamma} 1(\mathscr{A})
$$

shows that $\langle\alpha \supset \beta, \alpha\rangle \Rightarrow \beta, \alpha \supset \beta,\langle\langle\alpha\rangle \Rightarrow \beta\rangle \Rightarrow \gamma \vdash \gamma$. From the derivability of MP and Lemma 3.5 we obtain $\alpha \supset \beta,\langle\langle\alpha\rangle \Rightarrow \beta\rangle \Rightarrow \gamma \vdash \gamma$. Conversely, the derivation 
denoted by

$$
\frac{\overline{\alpha \supset \beta}^{(\mathscr{A})} \quad 1 \frac{\bar{\alpha}}{\beta}(\mathscr{A})}{\beta} 1(\mathscr{A})
$$

shows that $\langle\alpha \supset \beta,\langle\langle\alpha\rangle \Rightarrow \beta\rangle \Rightarrow \beta\rangle \Rightarrow \beta, \alpha \supset \beta, \alpha \vdash \beta$. From the derivability of $\supset \mathrm{E}$ and Lemma 3.5 we obtain $\alpha \supset \beta, \alpha \vdash \beta$.

One sees that $\vee \mathrm{E}$ is here considered to be the pattern according to which elimination rules are framed. To obtain an elimination rule for $\supset$ of this form the extension of the concept of assumption was necessary: Elimination rules are of level $l+1$ if the introduction rules are of level $l$; and in the case of $\supset$, the introduction rules are already of level 3 (cf. Schroeder-Heister [10]).

Definition 4.5. The rank $\operatorname{rk}(\alpha)$ of a formula $\alpha$ is defined as follows: $\operatorname{rk}(\alpha)=0$, if $\alpha$ is atomic; $\operatorname{rk}\left(S \alpha_{1} \cdots \alpha_{n}\right)=\max \left\{\operatorname{rk}(\beta) \mid\right.$ there is a schema $\Phi\left(A_{1}, \ldots, A_{n}\right) \Rightarrow S A_{1} \cdots A_{n}$ for $S$ I so that $\beta$ is an element of $\left.\Phi\left(\alpha_{1}, \ldots, \alpha_{n}\right)\right\}+1$.

That this is a correct inductive definition is due essentially to the requirement that the lists of rule schemata associated with $S$ contain only operators preceding $S$ in a certain enumeration of operators. $\operatorname{rk}(\alpha)$ is not simply defined to be the number of operators in $\alpha$, because one wants the rank to increase when an introduction rule is applied, and the formulas from which $\alpha$ is inferred by an introduction inference and rules discharged by this inference step may contain more and other operators than $\alpha$.

THEOREM 4.6 (REPLACEMENT THEOREM). Let $\beta$ t $\beta^{\prime}$. Let $\beta$ occur as a subformula of $\Gamma$ and let $\Gamma\left[\beta / \beta^{\prime}\right]$ be the result of replacing this occurrence of $\beta$ in $\Gamma$ by $\beta^{\prime}$. Then $\Gamma+\Gamma$ $\left[\beta / \beta^{\prime}\right]$.

PrOOF. It is sufficient to prove the theorem for formulas $\alpha$ instead of lists of rules $\Gamma$. The full assertion then follows from Lemma 3.8. We proceed by induction on $\operatorname{rk}(\alpha)$. If $\beta$ is identical with $\alpha$ (which must particularly be the case if $\operatorname{rk}(\alpha)=0$ ) the assertion is trivial. If $\alpha$ is $S \alpha_{1} \cdots \alpha_{i} \cdots \alpha_{n}$ and $\beta$ occurs as a subformula of $\alpha_{i}$ then for each $S$ I rule $\Phi\left(\alpha_{1}, \ldots, \alpha_{n}\right) \Rightarrow S \alpha_{1} \cdots \alpha_{n} \beta$ occurs (possibly more than once) as a subformula of elements of $\Phi\left(\alpha_{1}, \ldots, \alpha_{n}\right)$. Since these elements are of lower rank than $\alpha$, we obtain by the induction hypothesis and Lemma 3.8

$$
\Phi\left(\alpha_{1}, \ldots, \alpha_{i}\left[\beta / \beta^{\prime}\right], \ldots, \alpha_{n}\right) \text { ㄴ } \Phi\left(\alpha_{1}, \ldots, \alpha_{i}, \ldots, \alpha_{n}\right),
$$

and by Lemma 3.5 and $S I$

$$
\begin{gathered}
\Phi\left(\alpha_{1}, \ldots, \alpha_{i}\left[\beta / \beta^{\prime}\right], \ldots, \alpha_{n}\right) \vdash S \alpha_{1} \cdots \alpha_{n}, \\
\Phi\left(\alpha_{1}, \ldots, \alpha_{n}\right) \vdash S \alpha_{1} \cdots \alpha_{i}\left[\beta / \beta^{\prime}\right] \cdots \alpha_{n} .
\end{gathered}
$$

Application of $S E$ yields

$$
S \alpha_{1} \cdots \alpha_{n} \text { ㄴ } S \alpha_{1} \cdots \alpha_{i}\left[\beta / \beta^{\prime}\right] \cdots \alpha_{n} .
$$

In order to consider rules to be meaning-determining for logical operators, one may (as e.g. Belnap [1]) require fulfilment of two necessary conditions: uniqueness and noncreativity. This is particularly suggested by the interpretation of Condition 4.2 as a generalization of the aims of explicit definitions. Both requirements are fulfilled, as the following theorems show. 
THEOREM 4.7. Let the same lists of rule schemata be associated with the n-ary operators $S$ and $S^{\prime}$, i.e. let $S$ and $S^{\prime}$ have the same introduction and elimination rules aside from the naming of the operator. Let $\rho$ be a rule in which $S$ occurs at a certain place and let $\rho^{\prime}$ result from $\rho$ by replacing this occurrence of $S$ in $\rho$ by $S^{\prime}$. Then $\rho$ t $\rho^{\prime}$.

Proof. Combining $S$ I with $S^{\prime} E$ and $S^{\prime}$ I with $S E$ one obtains

$$
S \alpha_{1} \cdots \alpha_{n} \text { t } S^{\prime} \alpha_{1} \cdots \alpha_{n} \text { for arbitrary } \alpha_{1}, \ldots, \alpha_{n} .
$$

Now $S$ occurs in $\rho$ at the beginning of a certain subformula $S \beta_{1} \cdots \beta_{n}$ of an element of $\rho$. Replacing this $S \beta_{1} \cdots \beta_{n}$ by $S^{\prime} \beta_{1} \cdots \beta_{n}$ is the same as replacing $S$ by $S^{\prime}$. So the assertion follows from Theorem 4.6.

THEOREM 4.8. Let $S_{1}, S_{2}, \ldots$ be the enumeration of operators mentioned above Condition 4.2. Let $\Gamma \vdash_{i} \alpha$ mean that there is a derivation of $\alpha$ from $\Gamma$ using no basic rule for $S_{j}$ with $j>i$ (in the case $i=0$ no basic rule at all). Then for all $i \geq 1$, if $\Gamma \vdash_{i} \alpha$ and $S_{i}$ does not occur in $\Gamma$ nor in $\alpha$, then $\Gamma \vdash_{i-1} \alpha$.

In particular, if $\Gamma \vdash \alpha$ and $i$ is the greatest $j$ such that $S_{j}$ occurs in $\Gamma$ or $\alpha$, then $\Gamma \vdash_{i} \alpha$. In this case an $S_{j}$ with $j>i$ does not even occur in the derivation of $\alpha$ from $\Gamma$, i.e. $\alpha$ is derivable from $\Gamma$ in the calculus having only those $S_{j}$ with $j \leq i$ in its vocabulary.

This theorem states that the basic rules for $S_{i}$, when added to the basic rules for $S_{1}, \ldots, S_{i-1}$, do not create any derivability relation not involving $S_{i}$. The proof (which can be found in Schroeder-Heister [9]) proceeds mainly by modifying Prawitz's [7] normalization procedures and establishing normalizability for our generalized calculus.

In the theory of explicit definitions one usually requires besides noncreativity that defined terms can be eliminated. In the present context this would have to be formulated as follows: For each $\Delta$ there is a $\Delta^{\prime}$ containing no operators such that $\Delta+\Delta^{\prime}$. This is wrong, since it can be shown that no list of rule schemata $\Delta(A, B)$ exists such that, for all $\alpha$ and $\beta, \alpha \vee \beta \Vdash \Delta(\alpha, \beta)$ (cf. Schroeder-Heister [9]). Thus by extending the concept of explicit definability in Condition 4.2 we have retained noncreativity but given up eliminability. Eliminability fails in spite of the fact that uniqueness, in particular (5), holds. So there is no analogue to Beth's definability theorem for the present context.

§5. The completeness of $\{\&, \vee, \supset, 人\}$. Let $\mathscr{H}$ be a calculus of the form described in the last section containing among its operators the four standard connectives \&, $\vee, \supset$, $\curlywedge$ of intuitionistic sentential logic with the introduction and elimination rules given above. Let the first four operators in the enumeration $S_{1}, S_{2}, \ldots$ of the operators of $\mathscr{H}$ contain just these standard connectives. (This is possible because their introduction rule schemata have no operator in the premises.) Let $\mathscr{H}_{0}$ be the subcalculus of $\mathscr{H}$ having only $\&, \vee, \supset$, ᄉ as operators but allowing, as $\mathscr{H}$ does, assumption rules of arbitrary levels. Let $\mathscr{I}$ have only $\&, \vee, \supset, \wedge$ as operators, but MP instead of $\supset \mathrm{E}$ as basic rule schema; furthermore, in $\mathscr{I}$ only formulas are allowed as assumptions. So $\mathscr{I}$ can be considered to be a standard natural deduction system for intuitionistic logic. We show that each operator of $\mathscr{H}$ is explicitly definable in terms of $\&, \vee, \supset, \curlywedge$ and can therefore be expressed in $\mathscr{I}$.

Since for $\&$ and $\vee$ the associative laws hold, we omit brackets in iterated conjunctions and disjunctions. $\vdash$ always means $\vdash_{\mathscr{H}}$. 
We associate with each formula or formula schema $\alpha$, each rule or rule schema $\rho$ and each list of rules or of rule schemata $\Gamma$ a formula or formula schema $\alpha^{*}, \rho^{*}, \Gamma^{*}$ as follows:

Definition 5.1. $\alpha^{*}$ is $\alpha . \rho^{*}$ is $\left(\rho_{1}^{*} \& \cdots \& \rho_{n}^{*}\right) \supset \alpha$ if $\rho$ is $\left\langle\rho_{1}, \ldots, \rho_{n}\right\rangle \Rightarrow \alpha . \Gamma^{*}$ is $\rho_{1}^{*} \& \cdots \& \rho_{n}^{*}$ if $\Gamma$ is the list $\rho_{1} \cdots \rho_{n} . \Gamma^{*}$ is $\curlywedge \supset \curlywedge$ if $\Gamma$ is the empty list.

LEMMA 5.2. For all formulas $\alpha$, rules $\rho$, and lists of rules $\Gamma$ :

$$
\alpha \text { t } \alpha^{*}, \quad \rho \text { } \rho^{*}, \quad \Gamma \text { ㄴ } \Gamma^{*} .
$$

Proof. $\alpha$ t $\alpha^{*}$ holds by definition. From $\rho_{i}$ t $\rho_{i}^{*}$ for all $i(1 \leq i \leq n)$,

$$
\rho_{1}, \ldots, \rho_{n} \text { ㄴ } \rho_{1}^{*} \& \cdots \& \rho_{n}^{*}
$$

follows by use of $\& I$ and $\& E$, and from that

$$
\left\langle\rho_{1}, \ldots, \rho_{n}\right\rangle \Rightarrow \alpha \Vdash\left(\rho_{1}^{*} \& \cdots \& \rho_{n}^{*}\right) \supset \alpha
$$

follows by use of $\supset \mathrm{I}$ and $\supset$ E. So $\rho$ t $\rho^{*}$ is proved by induction on the level of $\rho$, and the third assertion follows from the second one if $\Gamma$ is nonempty. If $\Gamma$ is empty, $\Gamma$ ㅂ $\curlywedge \supset$ 人 is true according to our stipulations.

THEOREM 5.3 For each $n$-ary $S$ there is a formula schema $F\left(A_{1}, \ldots, A_{n}\right)$ containing at most \&, $\vee, \supset, \curlywedge$ as operators, such that for all formulas $\alpha_{1}, \ldots, \alpha_{n}$

$$
S \alpha_{1} \cdots \alpha_{n}+F\left(\alpha_{1}, \ldots, \alpha_{n}\right) \text {. }
$$

ProOF (by induction on $l$, where $S$ occurs in the $l$ th place of the enumeration of the operators). If $S$ is $S_{1}, S$ is one of the operators $\&, \vee, \supset$, ᄉ, and we can take $F$ to be $S A_{1} \cdots A_{n}$. Let $S$ be $S_{l}$ for $l>1$. If $S$ is a $\perp$-operator, let $F$ be $ᄉ$. Obviously $S \alpha_{1} \cdots \alpha_{n}$ ㅅ 人. Otherwise there are lists of rule schemata $\Phi_{i}\left(A_{1}, \ldots, A_{n}\right)(1 \leq i \leq m)$ associated with $S$. They contain at most the operators $S_{j}$ with $j<l$ whose numbers of argument places we denote by $n_{j}$. By the induction hypothesis, formula schemata $F_{j}\left(A_{1}, \ldots, A_{n j}\right)$ exist containing at most $\&, \vee, \supset, \curlywedge$ as operators so that for all formulas $\beta_{1}, \ldots, \beta_{n_{j}}$

$$
S_{j} \beta_{1} \cdots B_{n_{j}} \text { ㄴ } F_{j}\left(\beta_{1}, \ldots, \beta_{n_{j}}\right) .
$$

By successive application of Replacement Theorem 4.6 we obtain lists $\Phi_{i}^{\prime}\left(A_{1}, \ldots, A_{n}\right)$ $(1 \leq i \leq m)$ containing at most $\&, \vee, \supset$, $\curlywedge$ as operators so that for all formulas $\alpha_{1}, \ldots, \alpha_{n}$

$$
\Phi_{i}\left(\alpha_{1}, \ldots, \alpha_{n}\right) \Vdash \Phi_{i}^{\prime}\left(\alpha_{1}, \ldots \alpha_{n}\right) .
$$

Now take $F\left(A_{1}, \ldots, A_{n}\right)$ to be

$$
\left(\Phi_{1}^{\prime}\left(A_{1}, \ldots, A_{n}\right)\right)^{*} \vee \cdots \vee\left(\Phi_{m}^{\prime}\left(A_{1}, \ldots, A_{n}\right)\right)^{*} .
$$

Using Lemma 5.2, SI and $S E$ and $\vee \mathrm{I}$ and $\vee \mathrm{E}$, it can easily be shown that for all formulas $\alpha_{1}, \ldots, \alpha_{n}$

$$
S \alpha_{1} \cdots \alpha_{n} H\left(\Phi_{1}^{\prime}\left(\alpha_{1}, \ldots, \alpha_{n}\right)\right)^{*} \vee \cdots \vee\left(\Phi_{m}^{\prime}\left(\alpha_{1}, \ldots, \alpha_{n}\right)\right)^{*} .
$$

COROLLARY 5.4. For all rules $\rho$ there is a rule $\rho^{+}$containing at most $\&, \vee, \supset, \curlywedge$ as operators, so that $\rho$ t $\rho^{+}$.

Proof. This follows from Theorem 5.3 by successive applications of Replacement Theorem 4.6. 
THEOREM 5.5. For all rules $\rho_{1}, \ldots, \rho_{n}$ and formulas $\alpha$ there are formulas $\rho_{1}^{0}, \ldots, \rho_{n}^{0}$ and $\alpha^{0}$, containing at most $\&, \vee, \supset, \curlywedge$ as operators, so that

$$
\rho_{1}, \ldots, \rho_{n} \vdash_{\mathscr{H}} \alpha \quad \text { iff } \rho_{1}^{0}, \ldots, \rho_{n}^{0} \vdash_{\mathscr{I}} \alpha^{0} .
$$

Proof. Define $\rho^{0}$ to be $\rho^{+*}$, where ${ }^{+}$is taken from Corollary 5.4 and ${ }^{*}$ from Definition 5.1. Then

$$
\begin{array}{rlll}
\rho_{1}, \ldots, \rho_{n} \vdash_{\mathscr{H}} \alpha & \text { iff } & \rho_{1}^{+}, \ldots, \rho_{n}^{+} \vdash_{\mathscr{H}} \alpha^{+} & \text {(Corollary 5.4) } \\
\text { iff } & \rho_{1}^{0}, \ldots, \rho_{n}^{0} \vdash_{\mathscr{H}} \alpha^{0} & \text { (Lemma 5.2) } \\
\text { iff } & \rho_{1}^{0}, \ldots, \rho_{n}^{0} \vdash_{\mathscr{H}} \alpha^{0} & \text { (Theorem 4.8) } \\
\text { iff } & \rho_{1}^{0}, \ldots, \rho_{n}^{0} \vdash_{\mathscr{I}} \alpha^{0}, &
\end{array}
$$

since by Lemma $4.4 \supset \mathrm{E}$ can be replaced by MP and since no assumption rule of level $>1$ can have been applied within a derivation of a formula from assumption formulas alone provided the basic rules are all of level $\leq 3$.

COROLLARY 5.6. For all formulas $\alpha_{1}, \ldots, \alpha_{n}, \beta$, containing at most $\&, \vee, \supset, \curlywedge$ as operators:

$$
\alpha_{1}, \ldots, \alpha_{n} \vdash_{\mathscr{I}} \beta \text { iff } \alpha_{1}, \ldots, \alpha_{n} \vdash_{\mathscr{H}} \beta .
$$

Proof. $\alpha_{1}^{0}, \ldots, \alpha_{n}^{0}, \beta^{0}$ is the same as $\alpha_{1}, \ldots, \alpha_{n}, \beta$.

Theorem 5.5 shows that ${ }^{0}$ is an embedding of $\mathscr{H}$ in $\mathscr{I}$, and Corollary 5.6 shows that the identity function is an embedding of $\mathscr{I}$ in $\mathscr{H}$. In this sense $\mathscr{H}$ and $\mathscr{I}$ have equal expressive power. This result is of course relative to our framework that formulas formed by sentential operators have as their content the common content of lists of certain kinds of rules. So it should not be considered as justifying the restriction to $\&, \vee, \supset$, $\curlywedge$ in ordinary intuitionistic logic, but rather as demarcating the strength of these operators. This view, as similarly emphasized by Prawitz [8], avoids e.g. Kreisel's [4] rigorous criticism of the completeness results of McCullough [6] and Zucker and Tragesser [13].

$\{\&, \vee, \supset, \curlywedge\}$ is the most usual set of operators being complete in the sense of Theorem 5.3, but of course not the only one. Hendry [3] has shown that $\{\equiv, \vee, \curlywedge\}$ is a complete set, where the schema for $\equiv \mathrm{I}$ is of the form

$$
\langle\langle A\rangle \Rightarrow B,\langle B\rangle \Rightarrow A\rangle \Rightarrow(A \equiv B) \text {. }
$$

For all $\alpha, \beta$ it holds that

$$
\begin{aligned}
& \alpha \supset \beta+\beta \equiv(\alpha \vee \beta), \\
& \alpha \& \beta \text { ㄴ }(\alpha \equiv \beta) \equiv(\alpha \vee \beta) .
\end{aligned}
$$

If $s$ is the three-place operator with two schemata for $s \mathbf{I}$ of the form

$$
\langle\langle A\rangle \Rightarrow B,\langle B\rangle \Rightarrow A\rangle \Rightarrow s A B C, \quad\langle C\rangle \Rightarrow s A B C,
$$

then for all $\alpha, \beta, \gamma$ it holds that

$$
\alpha \equiv \beta \text { } \alpha \beta \beta \text { 人, } \quad \alpha \vee \beta \text { s (人三人 }) \alpha \beta,
$$

which shows that $\{s, \curlywedge\}$ is a complete set as well. Hendry has however shown that there is no binary operator $S$ such that $\{S\}$ is complete. 
§6. Comparison with related conceptions. Zucker and Tragesser [13] propose a so-called "inferential logic" based on the thesis of the primacy of introduction rules for a theory of meaning. They provide a standard form for introduction and elimination rules for operators having at most one introduction rule schema. Operators with more than one introduction rule schema are treated by the remark that they are definable from $\&, \vee, \supset, \curlywedge$. This definability claim is not quite clear, since it is based only on the implicit assumption that when an operator is given by more than one introduction rule schema, we obtain the meaning of the operator in terms of $\&, \vee, \supset$, $\curlywedge$ by forming the disjunction of the meanings of premises of introduction rule schemata in terms of $\&, \vee, \supset$, ᄉ.

In contrast to Zucker and Tragesser, Prawitz [8] provides a standard form for elimination rule schemata which is also suitable for operators with more than one introduction rule schema. Thus he is able to formally prove a result similar to Theorem 5.3. His semantic framework, developed within a theory of argumentation, differs from that of Zucker and Tragesser, and from the one given here, particularly in his justification of elimination rules. Prawitz uses $\supset$ as a basic sign in his standard form of elimination rule schemata, thus presupposing a concept of implication at a place where we use rules as assumptions. If one read assumption rules as a special kind of left-iterated implications (we have avoided this here, but it is possible), our standard form of basic rule schemata for operators would turn out closely related to Prawitz's.

Kutschera [5] uses a kind of calculus of sequents instead of a natural deduction formulation in which the sequent arrow $\rightarrow$ may be iterated (to the left and to the right) and where the succedent may be empty (in which case a rule of thinning is at our disposal). Furthermore he confines himself to sentential operators not referring to other operators in the premises of their introduction rule schemata and proves completeness for $\{\&, \vee, \supset, \neg\}$ instead of $\{\&, \vee, \supset, \curlywedge\}$. However, if we identify empty succedents with $\curlywedge$ and Kutschera's sequent arrow $\rightarrow$ with our $\Rightarrow$, understanding $\Delta \Rightarrow(\Gamma \Rightarrow \alpha)$ always to be an abbreviation for $\Delta, \Gamma \Rightarrow \alpha$, then each (possibly higher level) sequent of Kutschera's system is derivable in that system if it is derivable in our system.

Došen [2], who does not deal with the completeness of sets of operators, makes use of sequents of arbitrary levels to investigate logical systems with different assumptions about structural rules. Contrary to that, we have not questioned the structural assumptions underlying Gentzen-Prawitz-style natural deduction. Furthermore, Došen allows operators to be introduced in and eliminated from the antecedent of a sequent, which does not fit in with our framework of natural deduction. Aside from these differences, the aim of Došen's theory is quite distinct from ours. He is not concerned with questions of semantics or theories of meaning at all, but develops a criterion for the logicality of constants involved in alternative systems.

\section{REFERENCES}

$\rightarrow$ N. D. Belnap, Tonk, plonk and plink, Analysis, vol. 22 (1961-1962), pp. 130-134.

[2] K. DoŠEN, Logical constants. An essay in proof theory, D. Phil. Thesis, Oxford, 1980.

$\rightarrow$ H. E. HENDRY, Does IPC have a binary indigenous Sheffer function?, Notre Dame Journal of Formal Logic, vol. 22 (1981), pp. 183-186. 
[4] G. Kreisel, Constructivist approaches to logic, Modern logic-a survey (E. Agazzi, editor), Reidel, Dordrecht, 1981, pp. 67-91.

$\longleftrightarrow \rightarrow$ F. v. KuTSCHERA, Die Vollständigkeit des Operatorensystems. $\{\neg, \wedge, \vee, \supset\}$ für die intuitionistische Aussagenlogik im Rahmen der Gentzensemantik, Archiv für Mathematische Logik und Grundlagenforschung, vol. 11 (1968), pp. 3-16.

[6] D. P. MCCulLOUGH, Logical connectives for intuitionistic propositional logic, this JouRnaL, vol. 36 (1971), pp. 15-20.

[7] D. PRAwitz, Natural deduction. A proof-theoretical study, Almqvist \& Wiksell, Stockholm, 1965.

[8] - Proofs and the meaning and completeness of the logical constants, Essays on mathematical and philosophical logic (J. Hintikka et al., editors), Reidel, Dordrecht, 1979, pp. 25-40; slightly revised German translation in Conceptus, vol. 16 (1982), no. 38, pp. 31-44.

[9] P. SCHROEDER-HEISTER, Untersuchungen zur regellogischen Deutung von Aussagenverknüpfungen, Dissertation, Bonn, 1981.

[10] - Logische Konstanten und Regeln. Zur Deutung von Aussagenoperatoren, Conceptus, vol. 16 (1982), no. 38, pp. 45-59.

[11] - Generalized rules for quantifiers and the completeness of the intuitionistic operators \&, $\vee, \supset, \curlywedge, \forall, \exists$, Logic Colloquium '83 (M. M. Richter et al., editors), Lecture Notes in Mathematics, Springer-Verlag, Berlin (to appear).

[12] N. Tennant, Natural logic, Edinburgh University Press, Edinburgh, 1978.

[13] J. I. ZuCKeR and R. S. TRAGESSER, The adequacy problem for inferential logic, Journal of Philosophical Logic, vol. 7 (1978), pp. 501-516.

\author{
FACHGRUPPE PHILOSOPHIE \\ UNIVERSITÄT KONSTANZ \\ POSTFACH 5560 \\ 7750 KONSTANZ, FEDERAL REPUBLIC OF GERMANY
}

